\title{
Spreading effect of tDCS in individuals with attention-deficit/ hyperactivity disorder as shown by functional cortical networks: a randomized, double-blind, sham-controlled trial
}

\section{OPEN ACCESS}

Edited by:

Mauro Muszkat,

Federal University of São Paulo, Brazi

Reviewed by:

Rajshekhar Bipeta,

Gandhi Medical College and Hospital,

Hyderabad, India

Chi-Hung Juan,

National Central University, Taiwan

${ }^{*}$ Correspondence:

Camila Cosmo,

Neuromodulation Center, Spaulding

Rehabilitation Hospital, Harvard Medical School, 79/96 13th Street,

Charlestown, MA 02129, USA ccosmo@ufba.br

Specialty section:

This article was submitted to Child and Neurodevelopmental Psychiatry, a section of the journal Frontiers in Psychiatry

Received: 24 March 2015

Accepted: 20 July 2015

Published: 04 August 2015

Citation:

Cosmo C, Ferreira C, Miranda JGV, do Rosário RS, Baptista AF Montoya $P$ and de Sena EP (2015)

Spreading effect of $t D C S$ in individuals with attention-deficit/ hyperactivity disorder as shown by functional cortical networks: a randomized, double-blind, sham-controlled trial.

Front. Psychiatry 6:111. doi: 10.3389/fpsyt.2015.00111

\section{Camila Cosmo ${ }^{1,2,3,4 *}$, Cândida Ferreira ${ }^{5}$, José Garcia Vivas Miranda ${ }^{5}$, Raphael Silva do Rosário ${ }^{5}$, Abrahão Fontes Baptista ${ }^{4,6}$, Pedro Montoya ${ }^{7}$ and Eduardo Pondé de Sena ${ }^{1}$}

\footnotetext{
Postgraduate Program, Interactive Process of Organs and Systems, Federal University of Bahia, Salvador, Brazil, ${ }^{2}$ Neuromodulation Center, Spaulding Rehabilitation Hospital, Harvard Medical School, Boston, MA, USA, ${ }^{3}$ Bahia State Department of Health (SESAB), Salvador, Brazil, ${ }^{4}$ Functional Electrostimulation Laboratory, Biomorphology Department, Federal University of Bahia, Salvador, Brazil, ${ }^{5}$ Institute of Physics, Federal University of Bahia, Salvador, Brazil, ${ }^{6}$ Postgraduate Program in Medicine and Human Health, School of Medicine, Federal University of Bahia, Salvador, Brazil, ${ }^{7}$ Research Institute in Health Sciences (IUNICS-IdisPa), University of the Balearic Islands, Palma, Spain
}

Background: Transcranial direct current stimulation (tDCS) is known to modulate spontaneous neural network excitability. The cognitive improvement observed in previous trials raises the potential of this technique as a possible therapeutic tool for use in attentiondeficit/hyperactivity disorder (ADHD) population. However, to explore the potential of this technique as a treatment approach, the functional parameters of brain connectivity and the extent of its effects need to be more fully investigated.

Objective: The aim of this study was to investigate a functional cortical network (FCN) model based on electroencephalographic activity for studying the dynamic patterns of brain connectivity modulated by tDCS and the distribution of its effects in individuals with ADHD.

Methods: Sixty ADHD patients participated in a parallel, randomized, double-blind, sham-controlled trial. Individuals underwent a single session of sham or anodal tDCS at $1 \mathrm{~mA}$ of current intensity over the left dorsolateral prefrontal cortex for $20 \mathrm{~min}$. The acute effects of stimulation on brain connectivity were assessed using the FCN model based on electroencephalography activity.

Results: Comparing the weighted node degree within groups prior to and following the intervention, a statistically significant difference was found in the electrodes located on the target and correlated areas in the active group $(p<0.05)$, while no statistically significant results were found in the sham group ( $p \geq 0.05$; paired-sample Wilcoxon signed-rank test). 
Conclusion: Anodal tDCS increased functional brain connectivity in individuals with ADHD compared to data recorded in the baseline resting state. In addition, although some studies have suggested that the effects of tDCS are selective, the present findings show that its modulatory activity spreads. Further studies need to be performed to investigate the dynamic patterns and physiological mechanisms underlying the modulatory effects of tDCS.

Trial Registration: ClinicalTrials.gov NCT01968512.

Keywords: attention-deficit/hyperactivity disorder, functional cortical networks, transcranial direct current stimulation, spreading effect, dorsolateral prefrontal cortex

\section{Introduction}

Transcranial direct current stimulation (tDCS), a non-invasive brain stimulation technique, is known to modulate spontaneous neural network excitability (1-3). A weak electrical current modifies the neuronal resting membrane potential to increase or decrease cortical activity according to whether the polarity applied is anodal or cathodal $(1,4)$. The technique is safe, inexpensive, and simple to apply, and this positive profile increases its potential applicability in different neuropsychiatric disorders such as attention-deficit/hyperactivity disorder (ADHD) (5-8).

In a crossover design, Bloch et al. studied the response of 13 ADHD patients to a single session of high-frequency repetitive transcranial magnetic stimulation (rTMS) (9). An increase in attention score was found in the active group compared to the sham group (9). Although up to the present moment, no studies have been conducted on the effect of tDCS in an ADHD population, the cognitive improvement in executive functions such as inhibitory control and attention observed in previous trials reinforces the potential of this technique as a possible therapeutic tool for use in this population (10-14).

To explore the potential of this technique as a treatment approach, it is important to understand the mechanisms involved in its modulation of brain connectivity. Physiological aspects of brain modulation have been examined in studies conducted in healthy volunteers and in individuals with neuropsychiatric conditions (4, 15-18). Nitsche and Paulus investigated the effects of tDCS on the brain using transcranial magnetic stimulation (TMS) as a tool with which to assess cortical excitability according to changes in the motor evoked potentials (MEP) (19). Using the repeated measures technique, those investigators studied 12 healthy participants submitted to tDCS at $1 \mathrm{~mA}$ over the left motor cortex (M1), and reported that the amplitudes of the MEP increased, demonstrating an increase in cortical excitability in the stimulated area (19). In a double-blind crossover trial, patients with major depressive disorder were submitted to anodal tDCS over the left dorsolateral prefrontal cortex (DLPFC) for $20 \mathrm{~min}$ at $2 \mathrm{~mA}$ (20). Theta changes were observed in the medial frontal cortex area, suggesting that the effect of tDCS consisted of indirect rather than direct modulation on the stimulated area, the left DLPFC (20).

Although previous trials have investigated the distribution and physiological mechanisms of the modulatory effects of tDCS $(4,9$, $15,19-23)$, up to the present moment no studies have been conducted to assess brain connectivity following tDCS in individuals with $\mathrm{ADHD}$. In this present study, the objective was to use a functional cortical network (FCN) model based on electroencephalographic activity to study the dynamic patterns of brain connectivity modulated by tDCS in individuals with ADHD. The hypothesis to be tested was that a single session of anodal tDCS over the left DLPFC at a current intensity of $1 \mathrm{~mA}$ for $20 \mathrm{~min}$ increases cortical connectivity compared to a sham group in adults with ADHD.

\section{Methods}

\section{Participants}

Sixty individuals (35 males and 25 females) with ADHD (mean age \pm SD: $32.2 \pm 10.9$ years) participated in the present parallel, randomized, double-blind, sham-controlled trial. To be eligible for inclusion in the study, individuals had to have been diagnosed with ADHD as defined in the Diagnostic and Statistical Manual of Mental Disorders, fourth edition, revised (DSM-IVTR), with diagnosis confirmed through careful assessment by an experienced psychiatrist. Other inclusion criteria were: age 18-65 years and being capable of understanding and signing the informed consent form. The exclusion criteria consisted of the presence of major psychiatric disorders; cognitive impairment; use of psychoactive substances or alcohol abuse in the previous 12 months; or any contraindication to the use of tDCS such as the presence of a metallic implant in the head or an implanted medical device. Cognitive impairment was defined as a score $\leq 24$ in the Mini-Mental State Examination (MMSE) $(24,25)$. The Mini International Neuropsychiatric Interview Plus (MINI-Plus) and the adult ADHD Self-Report Scale-18 (ASRS-18), a valid scale based on DSM-IV criteria $(26,27)$, were the instruments used for screening. With respect to the ASRS-18 assessment tool, subjects had to have a minimum score of 18 points.

Initially, 73 individuals were recruited through advertisements on the Internet and in social networks, and by e-mails and letters sent to neurologists, psychiatrists, neuropsychiatric societies and associations. Following prescreening interviews conducted by email and over the telephone, 13 subjects were excluded because they failed to meet the inclusion criteria. As shown in Figure 1, the remaining 60 patients composed the final sample.

Participants were randomly allocated to receive active or sham stimulation on a 1:1 basis. A permuted-block method was applied, with gender and age as prognostic variables. Thirty subjects 


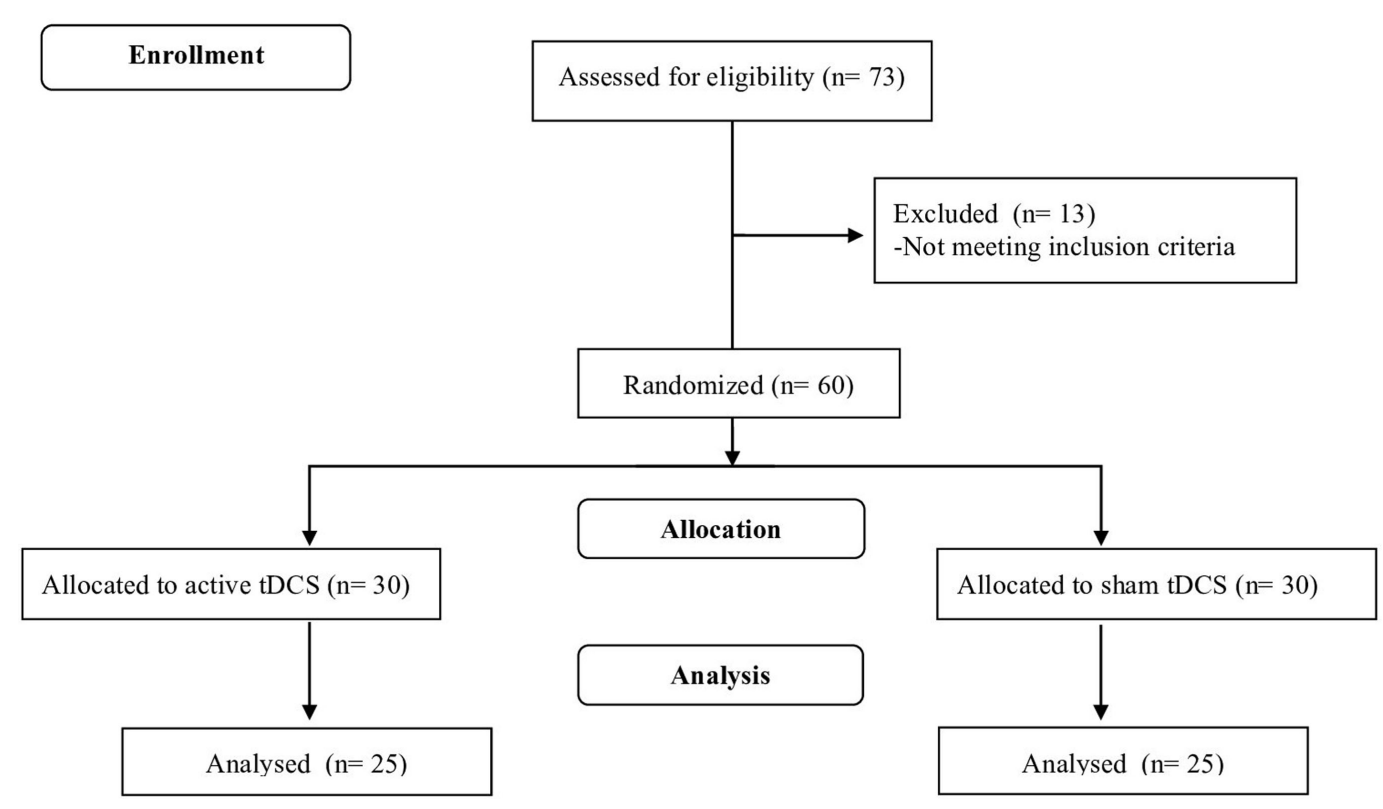

FIGURE 1 | Study flowchart adapted from CONSORT flow diagram.

were assigned to each group. An external investigator performed the randomization procedure, which was conducted using a computer-generated list of numbers (central randomization), with individuals being admitted to the study in the order of enrollment, thus guaranteeing the allocation concealment.

The institutional review board (IRB) of the Maternidade Climério de Oliveira, Federal University of Bahia approved the study protocol on October 9, 2012 (IRB number: 19311). All the participants gave their written informed consent after receiving detailed verbal and written information about the protocol in accordance with the ethical principles of the Declaration of Helsinki (28). The present study was conducted between May 2013 and April 2014 at the Laboratory of Functional Electrostimulation of the Federal University of Bahia (Salvador, Brazil).

\section{Procedures}

In all cases, the participation of the individuals in the study consisted of a single visit lasting $2 \mathrm{~h}$. Following the screening procedures, individuals were invited to sit in a comfortable chair in a sound-attenuated room before beginning the electroencephalogram (EEG) recording. During the first minute of the EEG recording, the participants were asked to look fixedly at a cross, after which recording took place over 4 min with the participant's eyes closed, in a resting state. The same EEG recording parameters were applied before and immediately following the interventions.

\section{Interventions}

The tDCS was applied using a Nemesys stimulator (Quark Medical Products, model Nemesys 941, Brazil). A single session of active or sham $\mathrm{tDCS}$ was performed in bi-frontal montage, placing the anodal electrode over F3 and the cathodal electrode at F4 (according to the international 10/20 EEG system), corresponding to the left and the right DLPFC area, respectively. A certified researcher administered the tDCS intervention. An electrical current of $1 \mathrm{~mA}$ was administered to the scalp through electrodes inserted in $35 \mathrm{~cm}^{2}$ saline-soaked sponges (current density $0.029 \mathrm{~mA} / \mathrm{cm}^{2}$ ) for $20 \mathrm{~min}$. To avoid discomfort, the current was ramped up over $30 \mathrm{~s}$ and ramped down over an equal interval of time at the end of the session (29). For sham stimulation, the current was applied over $30 \mathrm{~s}$ and subsequently turned off without the participant's knowledge to avoid he/she becoming aware of the group to which they had been allocated. This sham procedure mimics the initial perception of stimulation; however, without modulating brain excitability (30-32). To assess the success of the blinding approach, subjects were asked after the intervention whether they had received active or sham tDCS. Neither the participants nor the raters received information regarding which procedure had been applied (whether active or sham).

To assess safety, the participants were asked open-ended questions formulated in accordance with the tDCS adverse events questionnaire (33).

\section{Functional Cortical Network Model}

The FCN model based on EEG activity was applied to assess the acute effects of tDCS (34).

Electroencephalogram was recorded over a 5-min period prior to and following stimulation, totaling $10 \mathrm{~min}$, under two different conditions: $1 \mathrm{~min}$ with eyes open, looking fixedly at a cross, followed by $4 \mathrm{~min}$ with eyes closed in a resting state. Electroencephalographic recording was performed by applying 32 channels with the $\mathrm{Cz}$ electrode as a reference signal, using a BrainNet-BNT device (EMSA Medical Instruments, Brazil). EEG analysis was conducted using the EEGLAB/MATLAB software system (The Mathworks, Inc.). Cup electrodes $(\mathrm{Cu} / \mathrm{Au})$ were arranged following the international 10-20 system, with the following additional electrodes: FC3, FC4, CP3, CP4, FT7, FT8, TP7, TP8, and Oz. Electrode-skin impedance was set below $5 \mathrm{k} \Omega$. 
To perform the FCN, EEG data was converted from ASCII format to EEGLAB.set and the entire set of data was filtered between 0.5 and $50 \mathrm{~Hz}$. Artifacts were manually removed by visual inspection performed by an experienced investigator blinded to the intervention groups, using continuous artifact rejection. After these procedures, the final files were converted from.set to ASCII to develop the brain networks, using resting EEG data recorded before and after the intervention.

The FCN were constructed using a time-varying graph structure $(34,35)$ and then analyzing the EEG time series using a sliding time window. A correlation method, Motifs Synchronization, was applied to create a correlation matrix, $Q_{t}$ of $32 \times 32$ for each time window (36). A threshold criterion was used to make a matrix transformation to generate an adjacent matrix $A_{\mathrm{t}}$ with elements of 1 if the two electrodes were linked or 0 if they were independent. To carry on the weighted network, the Added Static Network (ASN) was used to obtain the sum of all adjacent matrices over the entire time interval, $\mathbf{T}$. For the weighted networks, the edge weights represent how many times this link has appeared over the time (Figure 2). Therefore, if $\left\{A_{\mathrm{t}}\right\}_{\mathrm{t}=1,2, \ldots, \mathrm{T}}$ is the set of adjacent matrices that represents the time-varying graphs $\left\{G_{\mathrm{t}}\right\}_{\mathrm{t}=1,2, \ldots, \mathrm{T}}$ then ASN is given by:

$$
S=\sum_{\mathrm{t}=1}^{\mathrm{T}} A_{\mathrm{t}}
$$

The outcome measure was the weighted node degree, as this parameter describes the network evolution. It corresponds to the number of links presented by a node over time. For the ASN, it is given as the number of times that an electrode was connected to any other in the network over the entire period, $T$, in which a timevarying graph was computed or the node degree was summed over time.

$$
k p_{i}=\sum_{\mathrm{t}=1}^{\mathrm{T}} k_{i_{t}}
$$

\section{FCN Analysis}

FCN analysis was performed using the EEGNET software system. Two resting state networks, pre and post stimuli, were performed for each participant by applying the time-varying graph together with the Motifs Synchronization method. For the purpose of analysis, a sliding time window of 20 points $(100 \mathrm{~ms})$, a motif degree of 3 , a lag interval of 1 , and $\tau=3$ were taken into consideration. A total of sixty ASN were computed for each intervention group.

\section{Statistical Analysis}

The clinical and demographic characteristics were assessed using descriptive statistical procedures such as measures of central tendency and dispersion. At baseline, these parameters were compared between the groups using the chi-square test for categorical variables and one-way analysis of variance (ANOVA) for continuous variables.

The Shapiro-Wilk test was used to assess the normality of data. Parameters of all the electrodes were analyzed for each individual and a weighted node degree was generated for each

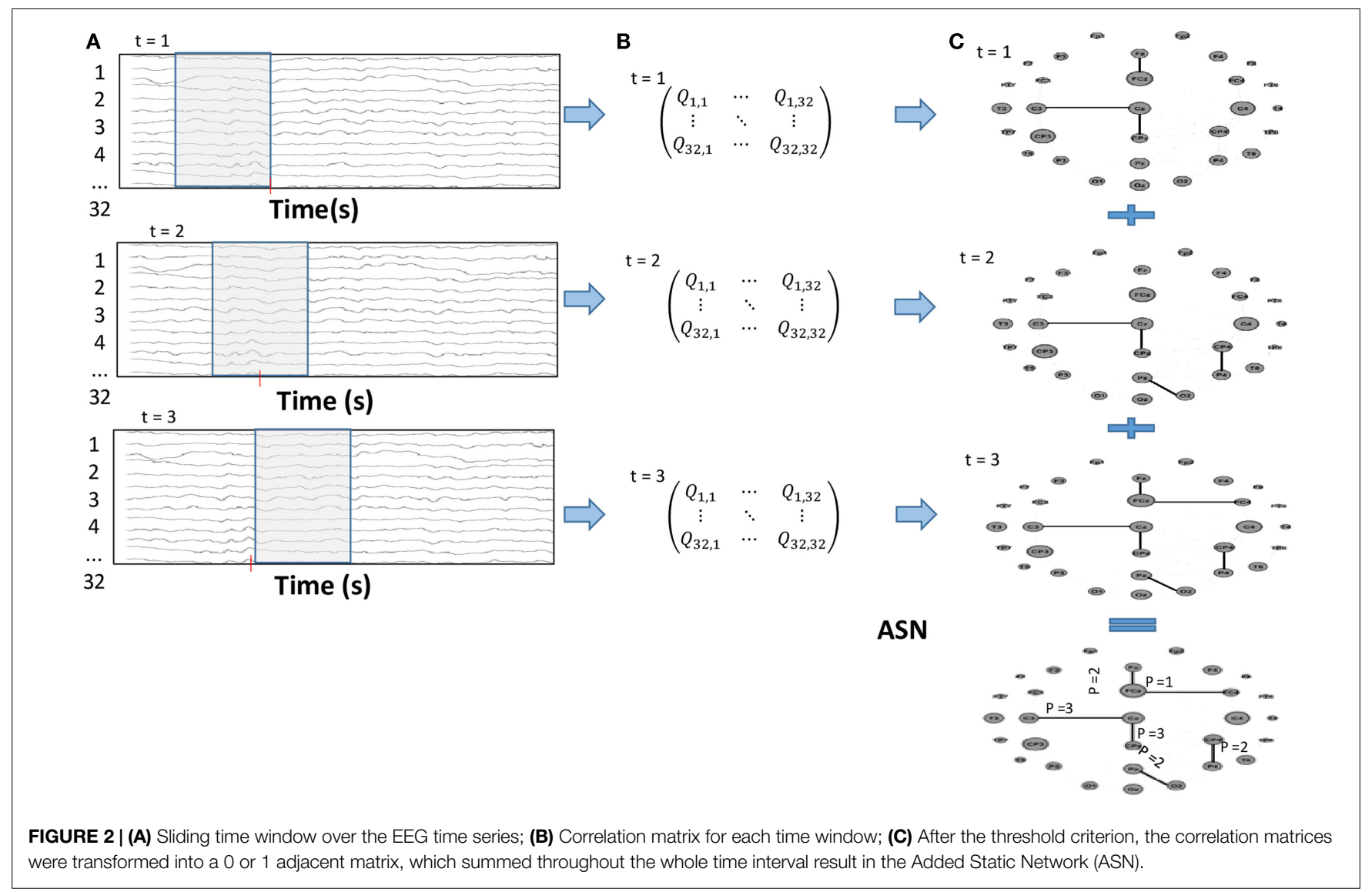


electrode per group, with $p$-values corrected using the Bonferroni technique. The outcome measure weighted node degree was analyzed applying the non-parametric two-sample Wilcoxon ranksum (Mann-Whitney) test. Additionally, changes in the outcome were determined as the difference between the weighted node degree after and before the intervention, with the resulting mean for each electrode being compared between groups using the same non-parametric approach. Wilcoxon paired test was used to compare pre- and post-intervention results within groups. Pearson's chi-square test was conducted to examine the effectiveness of the blinding procedure by assessing the interaction between patients' impressions of whether or not they had received the tDCS technique and what they had actually been given. Calculation of the sample size was based on previous studies using tDCS $(37,38)$, for a power of $80 \%$ and an alpha error of 0.05 . All the analyses were two-tailed. Predicting a dropout rate of $20 \%$, the final sample size was calculated at 60 individuals.

Statistical analyses were performed using the Stata software program, version 13.0 (StataCorp LP, College Station, TX, USA). Statistical significance was determined at $5 \%$ and all $p$-values were bidirectional.

\section{Results}

At baseline, no statistically significant differences were found between the active and sham groups for any of the demographic or clinical variables (Table 1). Although all the subjects completed the entire protocol, during the EEG/FCN analysis five participants were removed from each group, since, after artifact removal, fewer than $1200 \mathrm{~ms}$ of EEG recording remained. tDCS was well tolerated by all the participants and no adverse events or discomfort were reported.

Analyses of the weighted node degree prior to the interventions showed no significant differences between the active and sham groups $(U=335.00 ; p=0.86)$. Results were similar when the weighted node degree was assessed between the groups after the intervention $(U=319.00 ; p=0.92)$ (Figure 3). Furthermore, no significant differences were found between the groups (active vs. sham) when the changes in the weighted node degree (the difference between the post- and pre-intervention for each electrode) were analyzed ( $p \geq 0.05$; two-sample Wilcoxon rank-sum) (Table S1 in Supplementary Material).

When the weighted node degree was analyzed within the groups prior to and following the interventions, a significant difference was found in the active group with respect to the electrodes located on the stimulated area (left frontal area) as well as on the occipital, left and right temporal, and centroparietal areas $(p<0.05)$. In the sham group, on the other hand, no statistically significant difference was found ( $p \geq 0.05$; Paired-sample Wilcoxon signed-rank test) (Figure 4; Table S2 in Supplementary Material).

Regarding the blinding method, $43.33 \%$ of the participants submitted to active stimulation correctly guessed that they were in the active group, while $70 \%$ of the individuals in the sham group correctly reported that they had received sham tDCS. However, these differences were not statistically significant $\left(\chi^{2}=1.15\right.$; $p=0.28)$.
TABLE 1 | Demographic and clinical characteristics of subjects at baseline

\begin{tabular}{|c|c|c|}
\hline & $\begin{array}{l}\text { Active group } \\
n(30)\end{array}$ & $\begin{array}{l}\text { Sham group } \\
n(30)\end{array}$ \\
\hline Age (years) ${ }^{a}$ & $31.83(11.55)$ & $32.67(10.37)$ \\
\hline Males (\%) & 56.67 & 60.0 \\
\hline MMSE $^{\mathrm{b}}$ & $28.77(1.25)$ & $28.93(1.20)$ \\
\hline Mean duration of disease (years) & 21.77 & 22.90 \\
\hline \multicolumn{3}{|l|}{ Types of ADHD (\%) ${ }^{\mathrm{C}}$} \\
\hline Combined inattentive/hyperactive/impulsive & 76.67 & 70.00 \\
\hline Predominantly inattentive & 20.00 & 23.33 \\
\hline Predominantly hyperactive/impulsive & 3.33 & 6.67 \\
\hline
\end{tabular}

${ }^{a}$ Age presented as mean $\pm S D$.

${ }^{b}$ Mini-mental state examination (MMSE) described as mean $\pm S D$.

${ }^{C}$ According to the criteria of the Diagnostic and Statistical Manual of Mental Disorders, fourth edition, revised (DSM-IV-R).

$A D H D$, attention-deficit/hyperactivity disorder.

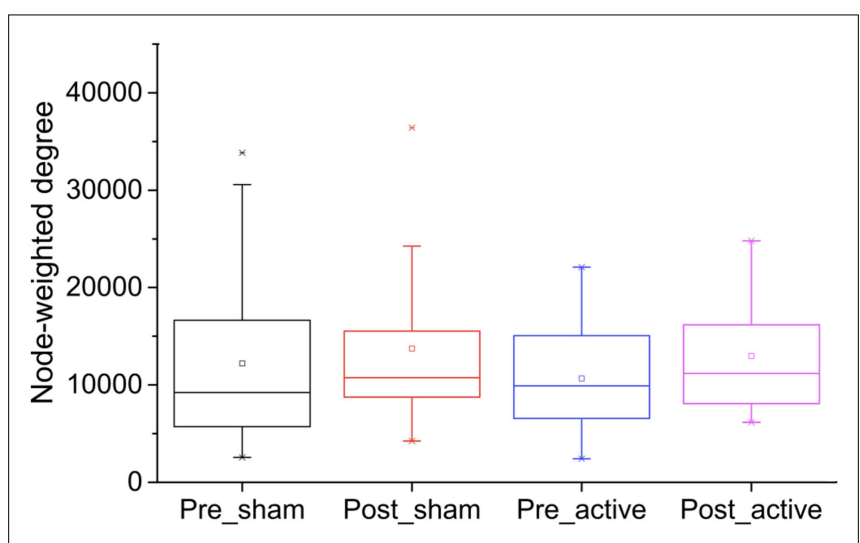

FIGURE 3 | Box plot representing the weighted node degree by group (active vs. sham) and time (pre vs. post intervention). No significant differences were found between the groups before and after the interventions $(p \geq 0.05)$

\section{Discussion}

Analysis of the weighted node degree showed a statistically significant difference in the active tDCS group between the preand post-intervention moments, while no statistically significant difference was found in the group submitted to sham stimulation. When the two groups were compared at the post-intervention moment, no differences were detected. These findings suggest that $\mathrm{tDCS}$ improves brain connectivity compared to the previous resting state values; however, in the present study, this increase was insufficient to enable differences to be detected between groups.

To the best of our knowledge, this is the first trial to apply a FCN model to examine the dynamic patterns of brain connectivity modulated by tDCS. The FCN model describes brain connectivity based on the correlation between the electrodes over time (34). Although tDCS has been widely studied and applied, its neurophysiological mechanisms remain to be fully clarified $(16-19,39,40)$. Therefore, the application of a new physiological measurement approach such as the FCN model presented here is important, since this is a feasible, inexpensive technique 


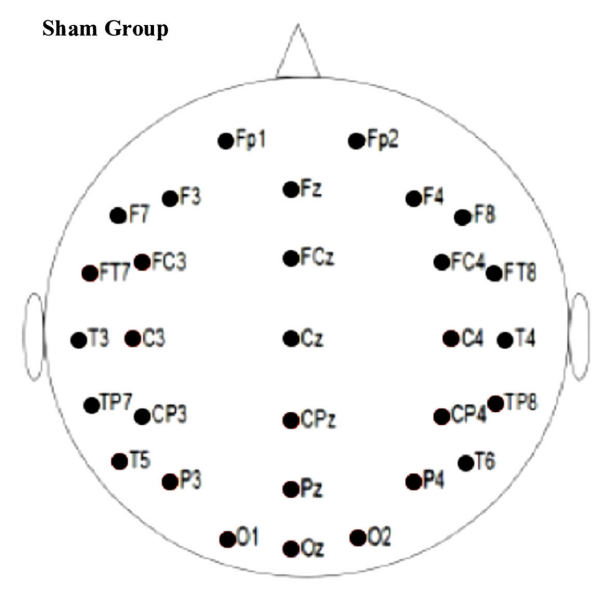

FIGURE 4 | Illustration of the result by electrode. The paired-sample Wilcoxon signed-rank test was used to compare the weighted node degree, pre- and post-intervention, within groups. A statistically

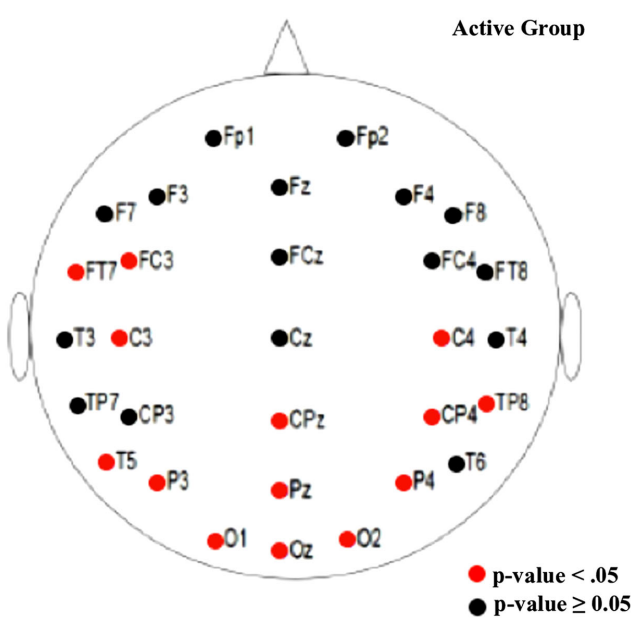

significant difference was observed in the active group $(p<0.05)$ while no statistically significant differences were found in the sham intervention $(p \geq 0.05)$. compared to neuroimaging or TMS. Furthermore, it allows the spatial changes in cortical connectivity induced by the modulatory activity of tDCS to be followed.

Recent studies have shown the importance of the analysis of temporal dynamics networks in electrophysiological investigation. Chu et al. and Betzel et al. reported the emergence of persistent functional connectivity between pairs of electrodes on a merged network, after summing the time of functional evolution into one single structure $(41,42)$. Likewise, it has been shown that global measures from evolving networks are different from random networks or those built from surrogate EEG data. Fraiman et al. found significant differences in local network indices for different perceptive behaviors using EEG temporal networks, revealing that cognitive patterns can be identified using time-varying methodology (34). Although all these findings suggest that this type of analysis constitutes a promising tool for the study of the dynamic patterns of cortical activity, the variability in the brain networks between individuals represents a challenge. This may be another explanation for the absence of any significant differences between the intervention groups. In addition, it also emphasizes the importance of paired tests applied in trials using the FCN model, as was done in the present study.

With respect to the paired analysis, an increase in cortical connectivity was found in the active group in the stimulated area, the left frontal area, as well as in the occipital, left and right temporal, and centroparietal areas. Although previous studies emphasized the selectivity of tDCS in the target area under the electrodes (22, 23, 43), the present findings appear to indicate diffuse effects. In a crossover sham-controlled study using EEG to measure the modulatory effects of tDCS, Jacobson et al. detected a localized decrease in theta activity following anodal stimulation over the right inferior frontal gyrus (22). In a trial conducted to investigate language production, Wirth et al. assessed the effects of tDCS using behavioral and electrophysiological parameters. Following anodal tDCS, an increase was found in cortical excitability as shown by a decrease in delta activity in the frontal stimulated area (23).

Despite the evidence reported from earlier studies regarding tDCS modulation of brain excitability in the stimulated area, some studies have proposed that tDCS may modify the neuronal activity of correlated brain areas far from the target region. In agreement with the present findings, Lauro et al. reported a local and diffuse modulation of cortical excitability following anodal stimulation (21). Applying TMS and electroencephalography techniques to examine how the effects of tDCS spread, those investigators demonstrated modulated neuronal connectivity in the target and contralateral hemispheres after $15 \mathrm{~min}$ of active stimulation with a current density of $0.08 \mathrm{~mA} / \mathrm{cm}^{2}$ (21). Modifications in cortical excitability were examined by evaluating cerebral blood flow in a study in which tDCS was applied over M1 (44). Compared to sham tDCS, anodal stimulation increased cerebral blood flow at M1 as well as in the contralateral cortical and subcortical areas, revealing the spread of the effects of tDCS (44). A crossover trial using functional magnetic resonance imaging (fMRI) measured the distribution of cortical excitability after $20 \mathrm{~min}$ of tDCS at $2 \mathrm{~mA}$ over the left DLPFC (45). Significant cortical activation was detected in the primary area of stimulation and in related brain networks such as the bilateral frontal-parietal and posterior cingulated cortex (45).

The spread of the effects of active stimulation observed in the present study, detected by analyzing the FCN, may be explained by the structural connections between the modulated areas, the left frontal, bilateral centroparietal, and occipital regions. Transcallosal modulation may also support the contralateral findings, as this brain structure is responsible for the majority of axonal connections between the hemispheres (46-48). 
Another relevant aspect refers to the effect of electrode location on these results. In the montage used in the present trial, the anodal electrode was placed over the left DLPFC while the cathodal electrode was placed over F4 (in accordance with the international 10/20 EEG system). The findings of the present study may have been the result of anodic activity, since the increase in brain excitability modulated by this stimulation may explain the higher connectivity between brain areas. As shown in Figure 4, excitability was most evident in the left frontal cortex (the stimulated area) and extended to the occipital, left and right temporal and centroparietal areas. In addition, the hyperpolarization promoted by the cathodal stimulation may explain the absence of increased connectivity over the whole right frontal cortex. These findings may support the hypothesis recently raised by Batsikadze et al. that at $1 \mathrm{~mA}$ (current density $0.029 \mathrm{~mA} / \mathrm{cm}^{2}$ ), anodal tDCS facilitates depolarization, while cathodal tDCS acts as an inhibitory electrode, decreasing cortical excitability, which was not found at a current intensity of $2 \mathrm{~mA}$ (49).

Comparison of the groups after the interventions revealed no statistically significant differences, which could be explained by the fact that the application consisted of a single session. Although we understand that multiple sessions of anodal tDCS could increase cortical connectivity, to date this is the first trial to investigate the effects of $\mathrm{tDCS}$ in an ADHD population. As this neurodevelopmental disorder involves alterations in brain excitability, with reduced cortical activation in prefrontal areas and increased activity in correlated areas, it was decided to opt for a more conservative approach, using a single session of tDCS to ensure safety while examining its effects in these patients.

As shown in previous studies using EEG and behavioral assessment, $\mathrm{tDCS}$ may improve executive functions $(10-14,37,50,51)$, including those affected in patients with ADHD such as inhibitory control and attention, thus suggesting that this technique may represent a possible therapeutic approach for this population. To evaluate the potential of tDCS, our initial focus was on understanding how it affects brain connectivity through the FCN. The aim of the present analysis was therefore to use this mathematical model to investigate the dynamic patterns of brain connectivity modulated by tDCS in patients with ADHD. Notwithstanding, these findings need to be interpreted with caution, since it is not behavioral parameters but, rather, neurophysiological results that are being presented. As mentioned above, this analysis reveals an increase in brain connectivity in the active group when baseline

\section{References}

1. Brunoni AR, Nitsche MA, Bolognini N, Bikson M, Wagner T, Merabet L, et al. Clinical research with transcranial direct current stimulation (tDCS): challenges and future directions. Brain Stimulat (2012) 5(3):175-95. doi:10. 1016/j.brs.2011.03.002

2. Nitsche MA, Paulus W. Excitability changes induced in the human motor cortex by weak transcranial direct current stimulation. J Physiol (2000) 527(Pt 3):633-9. doi:10.1111/j.1469-7793.2000.t01-1-00633.x

3. Kuo MF, Nitsche MA. Effects of transcranial electrical stimulation on cognition. Clin EEG Neurosci (2012) 43(3):192-9. doi:10.1177/1550059412444975

4. Kessler SK, Turkeltaub PE, Benson JG, Hamilton RH. Differences in the experience of active and sham transcranial direct current stimulation. Brain Stimulat (2012) 5(2):155-62. doi:10.1016/j.brs.2011.02.007 findings prior to tDCS are compared with results after tDCS, showing that this improvement is not limited to the target area but is also seen in correlated regions of the brain. Further studies and analyzes need to be performed to correlate these findings with behavioral assessments to evaluate whether or not higher cortical connectivity might be related to better cognitive performance.

\section{Conclusion}

The present findings suggest increased cortical connectivity, evidenced by a FCN model, in stimulated and related areas following active stimulation over the left DLPFC when compared to the baseline resting state in individuals with ADHD. Although the results of some studies have suggested that the effects of tDCS are selective $(22,23)$, the present findings and results from other studies show that the spread of the modulatory activity of tDCS, as well as the mechanisms underlying its effects, need to be more fully investigated.

\section{Author Contributions}

$\mathrm{CC}, \mathrm{AB}$, and $\mathrm{ES}$ conceived and designed the experiments. $\mathrm{CC}, \mathrm{RR}$, and ES performed the experiments. CC, CF, JM, RR, AB, PM, and ES analyzed and interpreted the data. CC, JM, and ES drafted the manuscript. CC, CF, JM, RR, AB, PM, and ES performed a critical review of the manuscript. All the authors read and approved the final version of the manuscript.

\section{Acknowledgments}

The authors are grateful to Professor Carlos Teles, Ph.D., who performed the randomization procedure and developed the allocation concealment approach for this trial. CC received a scholarship (BEX 12253-12-0) from the Coordination for the Improvement of Higher Education Personnel (CAPES), Brazilian Ministry of Education. PM received a scholarship (CAPES/CNPq 2947/2013) from CAPES and the Council for Scientific and Technological Development (CNPq), Brazilian Ministry of Education. JM also received a grant from CNPq 306998/2014-9.

\section{Supplementary Material}

The Supplementary Material for this article can be found online at http://journal.frontiersin.org/article/10.3389/fpsyt.2015.00111

5. Murphy DN, Boggio P, Fregni F. Transcranial direct current stimulation as a therapeutic tool for the treatment of major depression: insights from past and recent clinical studies. Curr Opin Psychiatry (2009) 22(3):306-11. doi:10.1097/ YCO.0b013e32832a133f

6. Nitsche MA, Boggio PS, Fregni F, Pascual-Leone A. Treatment of depression with transcranial direct current stimulation (tDCS): a review. Exp Neurol (2009) 219(1):14-9. doi:10.1016/j.expneurol.2009.03.038

7. Ferrucci R, Mameli F, Guidi I, Mrakic-Sposta S, Vergari M, Marceglia S, et al. Transcranial direct current stimulation improves recognition memory in Alzheimer disease. Neurology (2008) 71(7):493-8. doi:10.1212/01.wnl. 0000317060.43722.a3

8. Demirtas-Tatlidede A, Vahabzadeh-Hagh AM, Pascual-Leone A. Can noninvasive brain stimulation enhance cognition in neuropsychiatric disorders? Neuropharmacology (2013) 64:566-78. doi:10.1016/j.neuropharm.2012.06.020 
9. Bloch Y, Harel EV, Aviram S, Govezensky J, Ratzoni G, Levkovitz Y. Positive effects of repetitive transcranial magnetic stimulation on attention in ADHD subjects: a randomized controlled pilot study. World J Biol Psychiatry (2010) 11(5):755-8. doi:10.3109/15622975.2010.484466

10. Liang WK, Lo MT, Yang AC, Peng CK, Cheng SK, Tseng P, et al. Revealing the brain's adaptability and the transcranial direct current stimulation facilitating effect in inhibitory control by multiscale entropy. Neuroimage (2014) 90:218-34. doi:10.1016/j.neuroimage.2013.12.048

11. Juan $\mathrm{CH}$, Muggleton NG. Brain stimulation and inhibitory control. Brain Stimulat (2012) 5(2):63-9. doi:10.1016/j.brs.2012.03.012

12. Penolazzi B, Stramaccia DF, Braga M, Mondini S, Galfano G. Human memory retrieval and inhibitory control in the brain: beyond correlational evidence. J Neurosci (2014) 34(19):6606-10. doi:10.1523/JNEUROSCI.0349-14. 2014

13. Coffman BA, Clark VP, Parasuraman R. Battery powered thought: enhancement of attention, learning, and memory in healthy adults using transcranial direct current stimulation. Neuroimage (2014) 85(Pt 3):895-908. doi:10.1016/j. neuroimage.2013.07.083

14. Tanoue RT, Jones KT, Peterson DJ, Berryhill ME. Differential frontal involvement in shifts of internal and perceptual attention. Brain Stimulat (2013) 6(4):675-82. doi:10.1016/j.brs.2012.11.003

15. Schlaug G, Renga V, Nair D. Transcranial direct current stimulation in stroke recovery. Arch Neurol (2008) 65(12):1571-6. doi:10.1001/archneur.65.12.1571

16. Pellicciari MC, Brignani D, Miniussi C. Excitability modulation of the motor system induced by transcranial direct current stimulation: a multimodal approach. Neuroimage (2013) 83:569-80. doi:10.1016/j.neuroimage. 2013.06.076

17. Pena-Gomez C, Sala-Lonch R, Junque C, Clemente IC, Vidal D, Bargallo N, et al. Modulation of large-scale brain networks by transcranial direct current stimulation evidenced by resting-state functional MRI. Brain Stimulat (2012) 5(3):252-63. doi:10.1016/j.brs.2011.08.006

18. Zheng X, Alsop DC, Schlaug G. Effects of transcranial direct current stimulation (tDCS) on human regional cerebral blood flow. Neuroimage (2011) 58(1):26-33. doi:10.1016/j.neuroimage.2011.06.018

19. Nitsche MA, Paulus W. Sustained excitability elevations induced by transcranial DC motor cortex stimulation in humans. Neurology (2001) 57(10):1899-901. doi:10.1212/WNL.57.10.1899

20. Powell TY, Boonstra TW, Martin DM, Loo CK, Breakspear M. Modulation of cortical activity by transcranial direct current stimulation in patients with affective disorder. PLoS One (2014) 9(6):e98503. doi:10.1371/journal.pone.00 98503

21. Romero Lauro LJ, Rosanova M, Mattavelli G, Convento S, Pisoni A, Opitz A, et al. TDCS increases cortical excitability: direct evidence from TMS-EEG. Cortex (2014) 58:99-111. doi:10.1016/j.cortex.2014.05.003

22. Jacobson L, Ezra A, Berger U, Lavidor M. Modulating oscillatory brain activity correlates of behavioral inhibition using transcranial direct current stimulation. Clin Neurophysiol (2012) 123(5):979-84. doi:10.1016/j.clinph. 2011.09.016

23. Wirth M, Rahman RA, Kuenecke J, Koenig T, Horn H, Sommer W, et al. Effects of transcranial direct current stimulation (tDCS) on behaviour and electrophysiology of language production. Neuropsychologia (2011) 49(14):3989-98. doi:10.1016/j.neuropsychologia.2011.10.015

24. Folstein MF, Folstein SE, McHugh PR. "Mini-mental state". A practical method for grading the cognitive state of patients for the clinician. J Psychiatr Res (1975) 12(3):189-98. doi:10.1016/0022-3956(75)90026-6

25. Bertolucci PH, Brucki SM, Campacci SR, Juliano Y. [The mini-mental state examination in a general population: impact of educational status]. Arq Neuropsiquiatr (1994) 52(1):1-7. doi:10.1590/S0004-282X1994000100001

26. Kessler RC, Adler L, Ames M, Demler O, Faraone S, Hiripi E, et al. The world health organization adult ADHD self-report scale (ASRS): a short screening scale for use in the general population. Psychol Med (2005) 35(2):245-56. doi: 10.1017/S0033291704002892

27. Sheehan DV, Lecrubier Y, Sheehan KH, Amorim P, Janavs J, Weiller E, et al. The mini-international neuropsychiatric interview (M.I.N.I.): the development and validation of a structured diagnostic psychiatric interview for DSM-IV and ICD-10. J Clin Psychiatry (1998) 59(Suppl 20):22-33.

28. Carlson RV, Boyd KM, Webb DJ. The revision of the declaration of Helsinki: past, present and future. Br J Clin Pharmacol (2004) 57(6):695-713. doi:10.1111/ j.1365-2125.2004.02103.x
29. Accornero N, Capozza M, Pieroni L, Pro S, Davi L, Mecarelli O. EEG mean frequency changes in healthy subjects during prefrontal transcranial direct current stimulation. J Neurophysiol (2014) 112(6):1367-75. doi:10.1152/jn.000 88.2014

30. Gandiga PC, Hummel FC, Cohen LG. Transcranial DC stimulation (tDCS): a tool for double-blind sham-controlled clinical studies in brain stimulation. Clin Neurophysiol (2006) 117(4):845-50. doi:10.1016/j.clinph.2005.12.003

31. Lapenta OM, Minati L, Fregni F, Boggio PS. Je pense donc je fais: transcranial direct current stimulation modulates brain oscillations associated with motor imagery and movement observation. Front Hum Neurosci (2013) 7:256. doi:10. 3389/fnhum.2013.00256

32. Nitsche MA, Cohen LG, Wassermann EM, Priori A, Lang N, Antal A, et al. Transcranial direct current stimulation: state of the art 2008. Brain Stimulat (2008) 1(3):206-23. doi:10.1016/j.brs.2008.06.004

33. Brunoni AR, Amadera J, Berbel B, Volz MS, Rizzerio BG, Fregni F. A systematic review on reporting and assessment of adverse effects associated with transcranial direct current stimulation. Int J Neuropsychopharmacol (2011) 14(8):1133-45. doi:10.1017/S1461145710001690

34. Fraiman D, Saunier G, Martins EF, Vargas CD. Biological motion coding in the brain: analysis of visually driven EEG functional networks. PLoS One (2014) 9(1):e84612. doi:10.1371/journal.pone.0084612

35. Silva BBM, Miranda JGV, Corso G, Copelli M, Vasconcelos N, Ribeiro S, et al. Statistical characterization of an ensemble of functional neural networks. Eur Phys J B (2012) 85(10):9. doi:10.1140/epjb/e2012-30481-7

36. Olofsen E, Sleigh JW, Dahan A. Permutation entropy of the electroencephalogram: a measure of anaesthetic drug effect. Br J Anaesth (2008) 101(6):810-21. doi:10.1093/bja/aen290

37. Fecteau S, Knoch D, Fregni F, Sultani N, Boggio P, Pascual-Leone A. Diminishing risk-taking behavior by modulating activity in the prefrontal cortex: a direct current stimulation study. J Neurosci (2007) 27(46):12500-5. doi:10. 1523/JNEUROSCI.3283-07.2007

38. Loo CK, Alonzo A, Martin D, Mitchell PB, Galvez V, Sachdev P. Transcranial direct current stimulation for depression: 3-week, randomised, shamcontrolled trial. Br J Psychiatry (2012) 200(1):52-9. doi:10.1192/bjp.bp.111. 097634

39. Nitsche MA, Seeber A, Frommann K, Klein CC, Rochford C, Nitsche MS, et al. Modulating parameters of excitability during and after transcranial direct current stimulation of the human motor cortex. J Physiol (2005) 568(Pt 1):291-303. doi:10.1113/jphysiol.2005.092429

40. Li LM, Uehara K, Hanakawa T. The contribution of interindividual factors to variability of response in transcranial direct current stimulation studies. Front Cell Neurosci (2015) 9:181. doi:10.3389/fncel.2015.00181

41. Betzel RF, Erickson MA, Abell M, O’Donnell BF, Hetrick WP, Sporns O. Synchronization dynamics and evidence for a repertoire of network states in resting EEG. Front Comput Neurosci (2012) 6:74. doi:10.3389/fncom.2012.00074

42. Chu CJ, Kramer MA, Pathmanathan J, Bianchi MT, Westover MB, Wizon L, et al. Emergence of stable functional networks in long-term human electroencephalography. J Neurosci (2012) 32(8):2703-13. doi:10.1523/JNEUROSCI. 5669- 11.2012

43. Lang N, Nitsche MA, Paulus W, Rothwell JC, Lemon RN. Effects of transcranial direct current stimulation over the human motor cortex on corticospinal and transcallosal excitability. Exp Brain Res (2004) 156(4):439-43. doi:10.1007/ s00221-003-1800-2

44. Lang N, Siebner HR, Ward NS, Lee L, Nitsche MA, Paulus W, et al. How does transcranial DC stimulation of the primary motor cortex alter regional neuronal activity in the human brain? Eur J Neurosci (2005) 22(2):495-504. doi:10.1111/j.1460-9568.2005.04233.x

45. Keeser D, Meindl T, Bor J, Palm U, Pogarell O, Mulert C, et al. Prefrontal transcranial direct current stimulation changes connectivity of restingstate networks during fMRI. J Neurosci (2011) 31(43):15284-93. doi:10.1523/ JNEUROSCI.0542-11.2011

46. Tomasch J. Size, distribution, and number of fibres in the human corpus callosum. Anat Rec (1954) 119(1):119-35. doi:10.1002/ar.1091190109

47. Gazzaniga MS. Cerebral specialization and interhemispheric communication: does the corpus callosum enable the human condition? Brain (2000) 123(Pt 7):1293-326. doi:10.1093/brain/123.7.1293

48. Innocenti GM, Aggoun-Zouaoui D, Lehmann P. Cellular aspects of callosal connections and their development. Neuropsychologia (1995) 33(8):961-87. doi:10.1016/0028-3932(95)00033-Y 
49. Batsikadze G, Moliadze V, Paulus W, Kuo MF, Nitsche MA. Partially nonlinear stimulation intensity-dependent effects of direct current stimulation on motor cortex excitability in humans. J Physiol (2013) 591(Pt 7):1987-2000. doi:10.1113/jphysiol.2012.249730

50. Hsu TY, Tseng LY, Yu JX, Kuo WJ, Hung DL, Tzeng OJ, et al. Modulating inhibitory control with direct current stimulation of the superior medial frontal cortex. Neuroimage (2011) 56(4):2249-57. doi:10.1016/j.neuroimage. 2011.03.059

51. Hsu TY, Tseng P, Liang WK, Cheng SK, Juan CH. Transcranial direct current stimulation over right posterior parietal cortex changes prestimulus alpha oscillation in visual short-term memory task. Neuroimage (2014) 98:306-13. doi:10.1016/j.neuroimage.2014.04.069
Conflict of Interest Statement: The authors declare that the research was conducted in the absence of any commercial or financial relationships that could be construed as a potential conflict of interest.

Copyright (C) 2015 Cosmo, Ferreira, Miranda, do Rosário, Baptista, Montoya and de Sena. This is an open-access article distributed under the terms of the Creative Commons Attribution License (CC BY). The use, distribution or reproduction in other forums is permitted, provided the original author(s) or licensor are credited and that the original publication in this journal is cited, in accordance with accepted academic practice. No use, distribution or reproduction is permitted which does not comply with these terms. 